\title{
Increase Activity of Superoxide Dismutase in Adults Sprague- Dawley Rats Liver After Exposed To Bisphenol A
}

\author{
Ansoumane Kourouma ${ }^{\mathrm{a}^{*}}$, Duan Peng ${ }^{\mathrm{a}}$, Quan Chao ${ }^{\mathrm{a}}$, Yaima M. López Tamayo ${ }^{\mathrm{b}}$, \\ Liu Changjiang a, Wang Chengmin ${ }^{\mathrm{a}}$, Fu Wenjuan ${ }^{\mathrm{a}}$, Qi Suqin ${ }^{\mathrm{a}}$, Yu \\ Tingting ${ }^{\mathrm{a}}$, Yang Kedi ${ }^{\mathrm{a}^{*}}$ \\ ${ }^{a}$ Department of Occupational and Environmental Health, School of Public Health, Tongji Medical College, \\ Huazhong University of Science and Technology, 13 Hangkong Road, Wuhan 430030,Hubei, PR China. \\ ${ }^{b}$ Universidad de las Ciencias Informáticas (UCI), Carretera a San Antonio, Km2 1/2 CP :19370, La Habana, \\ Cuba.
}

*Corresponding Authors. E-mail: kourouma_00@yahoo.fr/yangkd@mails.tjmu.edu.cn

\begin{abstract}
Bisphenol A (BPA) has been reported to process hepatic toxicity. We investigated the hypothesis that $B P A$ can induce reactive oxygen species (ROS) by increasing oxidative stress in the liver. The dosing solutions were prepared by thoroughly and uniformly mixing BPA in corn oil at $0,2,10,50 \mathrm{mg} / \mathrm{kg}$ body weight/day, were administered intraperitoneally every forty-eight hours for 20 days to adults Sprague-Dawley rats. After $24 \mathrm{~h}$ of the last treatment, rats were weighed, sacrificed and organs harvested for analysis. The body weight of treated rats did not show significant change as compared with the corresponding control groups. In BPA treated rats there was a significant decrease in the weight of liver organ. Suggesting that there was not a linear relationship between liver weight and body weight during the treatment, suggesting that comparisons made in terms of relative organ weights do not necessarily take proper account of differences in body weight. The activity of antioxidant enzyme superoxide dismutase (SODs) in liver tissue significantly increased in all groups treated with BPA when compared to the control group suggesting uncontrolled overproduction of ROS and failure in antioxidant system. The results indicated that BPA induces oxidative stress in the liver of rats by increasing activity of SOD. These findings provide a possible toxicological evidence of an adverse effect of BPA on liver damage.
\end{abstract}

Keywords: Bisphenol A (BPA, 2, 2-bis (4-hidroxyphenyl) propane); Liver, Oxidative stress; ROS; SOD.

\section{Introduction}

Bisphenol A (BPA; 2, 2-bis-(4-hydroxyphenol)-propane) is a monomer used in plastic and food can liner's manufacture ${ }^{1}$. BPA has a weak estrogenic activity ${ }^{2}$ and has been implicated as an endocrine disrupter in the balance of the oxidant and antioxidant system. Antioxidants are scavengers by preventing cell and tissue damage that could lead to cellular damage and disease ${ }^{3}$. Reactive oxygen species (ROS) are cytotoxic agents causing damage by attacking cell membrane and $\mathrm{DNA}^{4}$. BPA is known to oxidative stress by affecting the redox status in the exposed organs ${ }^{5}$. There are some studies suggesting that BPA caused tissue injury in the liver, Kidney, brain, and other organs by leading to formation of $\operatorname{ROS}^{4,6,7}$; and also to accumulate in many tissues such as liver after high dose treatments to male rats ${ }^{8}$. Reactive $\mathrm{O}_{2}$ species (ROS) are produced in both unstressed and stressed cells. However, the defense system, when presented with increased ROS formation under stress conditions, can be overshoot. Molecular damage from ROS, a common pathway to different toxicants, is important in the pathogenesis of toxic liver injury; a critical determinant of cellular defense against toxic outrage to the liver is the major scavenger of mitochondrial superoxide ${ }^{9}$. The liver has a range of antioxidant defense system. Among the well-known antioxidant enzymes protecting cells from ROS are the superoxide dismutases (SOD). There are three isoforms of SOD in mammalian cells: Cu/ZnSOD (SOD1), MnSOD (SOD2) and extracellular superoxide dismutase (esSOD or SOD3). In most tissues, the copper/zinc superoxide dismutase (Cu/ZnSOD or SOD1), a cytoplasmic copper-containing enzyme, is predominant ${ }^{10}$.SODs are clearly among the most important of those defenses, when coupled with the necessary downstream events for full detoxification of ROS. SODs specifically metabolize oxygen-free radicals and are believed to be the first and one of the most important lines of antioxidant enzyme defense systems against ROS. It catalyses the dismutation of superoxide into hydrogen peroxide $\left(\mathrm{H}_{2} \mathrm{O}_{2}\right)$ and oxygen, thus maintaining low steady-state levels of superoxide. Because excess superoxide is toxic, SOD is ubiquitously present in different organelles within the cells ${ }^{11}$. The present study was undertaken to analyse the possible association of BPA effect on the activity of SODs in rat SD liver. BPA may affect biological metabolism by disturbing redox control systems. Because the liver is the main organ of oxidative and detoxifying process as well as free radical reactions; in many diseases, biomarkers of oxidative stress are elevated in the liver at an early stage ${ }^{12}$. The findings of the study suggest that 
oxidative stress starts as early onset of diabetes mellitus, hypertension and cardiovascular diseases and increases progressively. The present study was undertaken to analyse the possible association of BPA effect on the activity of SODs in rat SD liver.

\section{Animals}

\section{Material And Methods}

Twenty four healthy male Sprague-Dawley rats (50-days olds, weighing 170-185 g) were purchased from the Tongji Medical College Animal Laboratory (Wuhan, China) and kept in accordance with the Guide for the Care and Use of Laboratory Animals published by Ministry of Health of People's Republic of China (Permit Number: 2011-s2456).

\section{Treatments}

The animals were housed in plastic cages under a well-regulated light and dark schedule ( $12 \mathrm{~h}$ light: 12 $\mathrm{h}$ dark) at $24 \pm 3^{\circ} \mathrm{C}$, humidity $(50 \pm 5 \%)$ environment, and free access to chow and tap water ad libitum. The rats were randomly divided into four groups, each group containing six rats. Each group (list the groups e.g., control group, low dose group, middle dose group and high dose group) was fed different doses of bisphenol A $0,2,10$, $50 \mathrm{mg} / \mathrm{kg}$ body weight respectively in corn oil every forty-eight hours by intra-peritoneal injection for 20 days. After 20-days of treatment, the rats were sacrificed. Ethical clearance for the use of animals in the study was obtained from the Institutional Animal Ethics Committee prior to the initiation of the study, and the experiments were performed in accordance with the guidelines for the Care and Use of Laboratory Animals published by Ministry of Health of People's Republic of China.

\section{Dose selection and preparation}

The doses and time used for the present study were derived from published data ${ }^{13,14}$ and the results of our preliminary experiment. BPA was dissolved in corn oil to obtain the desired concentration of BPA dose range, i.e., $0,2,10$ and $5 \mathrm{mg} / \mathrm{kg}$. An additional control group that had received only corn oil. Dose formulations were mixing well and stored in crystal bottles at $37^{\circ} \mathrm{C}$ overnight and were subsequently kept at room temperature throughout the study. Solutions were mixed thoroughly before use.

\section{Chemicals and reagents}

Bisphenol A (2,2-Di (4-hidroxyphenyl) propane) was purchased from (DR Co., Augsburg, Germany, purity: 98.5\%). Corn oil was obtained from (Sigma-Aldrich, St. Louis, MO, USA). Sigma Chemical Co. (St Louis, MO) USA, Collagenase, Trypsin-EDTA were obtained from GIBCO (Grand Island, NY, USA), Sodium lauryl sulphate from SRL, Eosin stain, Hematoxylin stain, Orange G stain from HiMedia (Mumbai). GSH-Px, MDA and SOD assay kit (Jiancheng Bioengineering Ltd., Nanjing, China).

\section{Body weight and organ collection}

The weight of each animal was recorded every forty-eight hours and any gross abnormality was noted. The animals were fasted overnight, weighed and killed by cervical dislocation. Liver and other organs were isolated from adhering tissues and weighed independently. The liver was quickly frozen at $-70{ }^{\circ} \mathrm{C}$ for later use for biochemical assays.

\section{SOD analysis}

The liver was homogenized using lysis buffer (containing $1 \mathrm{mM} \mathrm{Na}_{2} \mathrm{EDTA}, 150 \mathrm{mM} \mathrm{NaCl}, 10 \mathrm{mM}$ PMSF, $10 \mathrm{mM}$ Tris, $1 \mathrm{mM}$ aprotin) to evaluate oxidase stress following the protocol of SOD assay kit (Jiancheng Bioengineering Ltd., Nanjing, China).

- SOD activity in supernatant was determined by determining the reduction of nitro blue tetrazolium (NBT) by $\mathrm{O}^{2-}$ produced from the xanthine-xanthineoxiase system. One unit of SOD was defined as the amount protein inhibits the rate of NBT reduction by $50 \%$. Results were defined as U/mg protein.

\section{Statistical analysis}

Data are presented as the Mean \pm S.E.M. and were analyzed using the GraphPad PrismTM software version 5.0 (San Diego, USA) and SPSS statistical package 17.0 (SPSS Inc, Chicago, IL, USA). Comparison of means for treatment and control groups were done by independent-Sample T-test. The data were presented as mean \pm S.D. for six animals per group.

\section{Results}

Table 1 shows the body weight changes in rats administered with the BPA. The rats received the BPA consistently gain weight during the period of study, while the control group that only received corn oil gained 
weight. So no significant difference of bisphenol A-treated rats compared with the corresponding control groups $(\mathrm{P}<0.05)$.

Table 1: Body weight gains ( $\mathrm{g}$ ) by rat after administration of BPA in corn oil

\begin{tabular}{|c|c|c|c|c|}
\hline \multirow[b]{2}{*}{ Day } & \multicolumn{2}{|r|}{ Treatment } & \multicolumn{2}{|l|}{ groups } \\
\hline & Control & Low 2mg/kg (n=6) & Middle 10mg/kg $(\mathrm{n}=6)$ & High $50 \mathrm{mg} / \mathrm{kg}(\mathrm{n}=6)$ \\
\hline 1 & $183.69 \pm 14.37$ & $176.87 \pm 10.43$ & $185.50 \pm 10.20$ & $196.66 \pm 15.08$ \\
\hline 2 & $192.26 \pm 14.07$ & $185.79 \pm 5.11$ & $196.40 \pm 11.15$ & $196.66 \pm 13.75$ \\
\hline 3 & $198.47 \pm 15.28$ & $188.04 \pm 10.01$ & $196.93 \pm 13.31$ & $198.96 \pm 15.63$ \\
\hline 4 & $210.22 \pm 15.89$ & $196.08 \pm 10.65$ & $207.81 \pm 14.45$ & $207.96 \pm 15.96$ \\
\hline 5 & $218.72 \pm 17.5$ & $203.46 \pm 10.55$ & $213.91 \pm 15.85$ & $216.82 \pm 15.81$ \\
\hline 6 & $221.13 \pm 18.78$ & $206.66 \pm 9.36$ & $216.94 \pm 19.09$ & $220.52 \pm 14.50$ \\
\hline 7 & $225.81 \pm 18.66$ & $209.81 \pm 10.83$ & $220.67 \pm 18.24$ & $226.51 \pm 15.48$ \\
\hline 8 & $225.14 \pm 17.49$ & $208.53 \pm 9.56$ & $219.31 \pm 19.41$ & $229.83 \pm 17.20$ \\
\hline 9 & $242.11 \pm 17.35$ & $216.79 \pm 11.65$ & $229.16 \pm 20.64$ & $235.45 \pm 17.98$ \\
\hline 10 & $245.54 \pm 18.29$ & $218.74 \pm 12.89$ & $229.36 \pm 18.79$ & $236.92 \pm 17.36$ \\
\hline Last day & $237.06 \pm 19.67$ & $206.60 \pm 12.65$ & $218.84 \pm 19.41$ & $224.95 \pm 17.82$ \\
\hline
\end{tabular}

Effect of BPA on body weight of adult SD rats at every forty-eight hours for 20 days. Data represent as means \pm S.E.M.( $n=6$ rats per group).

\section{Liver weight}

As shown in Figures 1, have significant difference in absolute and relative weight of rat liver between control groups. The weights of the liver, decreased significantly when we gradually increased the concentration of BPA to $50 \mathrm{mg} / \mathrm{kg},(\mathrm{P}<0.05)$.

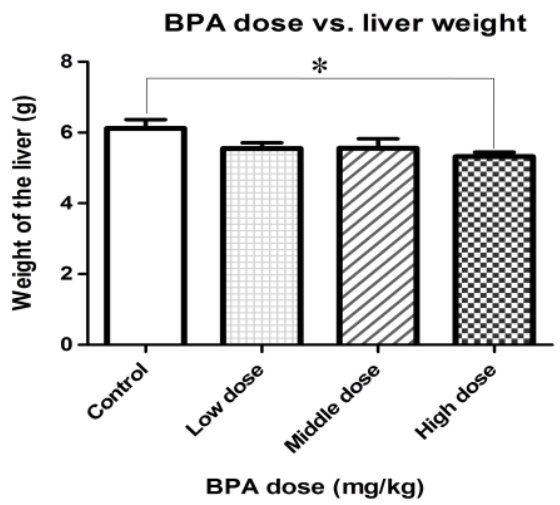

Figure 1. Effect of BPA on weight of the liver of adult SD rats. Data represent as means \pm S.E.M.(n=6 rats per group). ${ }^{*} \mathrm{P}<0.05$ denotes significant difference compared with controls.

Among the BPA treated rats, the activities of superoxide dismutase (SOD) increased significantly (**P $<0.01, * \mathrm{P}<0.05$, Figure 2) respectively a dose dependent, was observed in response to BPA treatment when compared with the control group $(* * \mathrm{P}<0.01$, Table 1).

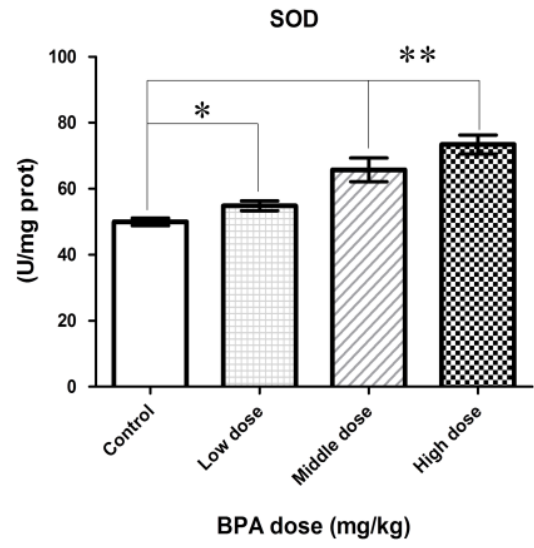

Figure 2. The Effect of BPA on SOD activity in the adult SD rat liver . BPA (0, 2, 10 and $50 \mathrm{mg} / \mathrm{kg} / \mathrm{day}) \mathrm{was}$ administrated ip. every forty-eight hours for 20 days. After the last administration, the rats were sacrificed decapitation and liver tissue was carefully dissected and stored at $-70{ }^{\circ} \mathrm{C}$ until analyzed. Data represent as means \pm S.E.M. ( $\mathrm{n}=6$ rats per group). $* \mathrm{P}<0.05$ denotes significant difference compared with controls. 


\section{Discussion}

The present research aimed to evaluate whether exposure to BPA induces oxidative stress in the liver of adults male rats. BPA has been demonstrated in both in vivo and in vitro experiments to acts endocrine disrupting chemicals released in the environment ${ }^{15}$. In our examination, the SODs activities in the liver tissue increased after administration of BPA. This result may be that SODs usually dismutases the superoxide anion radical into hydrogen peroxide which is degraded by catalase using reduced glutathione. Reduction in the activity of catalase may reflect inability of liver to eliminate hydrogen peroxide after exposure to BPA ${ }^{6}$. SODs are an important antioxidant enzyme which rapidly catalyzes the dismutation of superoxide anion $\left(\mathrm{O}^{-}\right)$and thus acts as a first line antioxidant defense. In the case of SOD deficiency or increased superoxide production, it reacts with nitric oxide to produce peroxynitrite $\left(\mathrm{ONOO}^{-}\right)$, which is potent oxidant and nitrosating agent that can cause direct damage to proteins, lipids, and DNA ${ }^{16}$.

The monitoring of body weight and organ weight reference values at each testing facility for laboratory animals used in toxicological studies has become a standard practice ${ }^{17}$. Weighing of the liver tissue of treated animals may reveal specific changes against to the control group (Figure 2). The issue of the effect of graded doses of BPA on weights of the rat liver is controversial. The present study was carried out to contribute to the discussion on this controversy. In the present study, the animals were administered BPA for 20 days in order to evaluate its. There were no significant differences in absolute body weight of BPA treated groups when compared to the control group $(\mathbf{P}<\mathbf{0 . 0 5})$ (Table 1). Our study found that there was not a linear relationship between liver weight and body weight during the treatment, suggesting that comparisons made in terms of relative organ weights do not necessarily take proper account of differences in body weight. But it necessary the need great care in evaluating the direct effect of BPA on liver weight when rapid changes in body weight are also occurring.

However, the tissues (liver) antioxidant enzymes evaluation seems to have important role in the etiology of ROS. The activity of total SOD increased significantly in liver, was observed in response to BPA treatment when compared with the control group (Figure 2). The increase in the activity of SOD may be due to higher enzyme activity, but do not mean better antioxidative protection. This increase may be due to its induction by increased production of superoxide $\left(\mathrm{O}_{2}{ }^{-}\right)$, which has been implicated in cell dysfunction ${ }^{3}$. Study has been reported that SOD levels were increased in the serum of patients with liver disease and diabetes than in normal subjects ${ }^{18}$. In addition, increased superoxide activity has been shown to play an important part in the pathogenesis of different genetic and acquired forms of hypertension in experimental animals, such as, spontaneously hypertensive rats ${ }^{19-21}$, angiosensin-infused rats ${ }^{22,23}$, salt-sensitive Dahl rats ${ }^{24}$ and lead-exposed rats ${ }^{25}$. On the other hand over expression of SOD may reflect a defect in the development or maturation of spermatozoa, as well as sperm cellular damage, resulting in decreased sperm fertilization potential ${ }^{26,27}$; knowing that spermatozoa are avoid of cytoplasmic enzyme systems that are required to repair the damage induced by oxidative stress ${ }^{28}$.

SOD-2 is located in mitochondria and belongs to a family superoxide dismutase enzymes converts superoxide radicals to $\mathrm{H}_{2} \mathrm{O}_{2}$, which is subsequently converted by catalase or glutathione peroxidase to $\mathrm{H}_{2} \mathrm{O}^{29-31}$. In our study SOD activity increased in the liver tissue. Therefore, these findings suggest that the administration of BPA induces overproduction of $\mathrm{H}_{2} \mathrm{O}_{2}$ in the liver. BPA also induces ROS and disrupt the mitochondria membrane resulting in release of cytochrome-C protein from the mitochondria that activates the caspases and induce apoptosis ${ }^{32}$. Meanwhile, the mechanisms by which SOD-2 can led to increased cell death have been reported ${ }^{30,31,33,34}$. Also another mechanism for SOD-2 induced apoptosis is through its ability to active p53 by production of $\mathrm{H}_{2} \mathrm{O}_{2}{ }^{35}$. The majority of reported BPA toxicities studies in literature have focused on reproductive effects these chemicals ${ }^{7,36,37}$. Besides their inherent effects on endocrine system ${ }^{38}$, and also known to inflict oxidative stress by affecting vital organs in humans ${ }^{39}$. In the present results including the oxidative stress markers (SODs) in the liver tissues and the decrease in relative liver weight of BPA administration could be caused by increased by ROS in liver, but this should be confirmed by histopathological study.

\section{Conclusions}

The results of our studies suggest that the observed changes in the level of SOD in the liver of rat, mat result from the occurrence of free radicals after administration of BPA. Our study reveals that graded doses of BPA generate ROS by increasing the activity of SOD thereby causing oxidative stress in liver of rats.

\section{Acknowledgements}

The authors thank the staff of Department of Occupational and Environmental Health and Ministry of Education Key Lab for Environmental and Health, School of Public Health, (Tongji Medical College) Huazhong University of Science and Technology, People's Republic of China, for providing various resources. 


\section{References}

[1]. Willhite, C. C., Ball, G. L. \& McLellan, C. J. Derivation of a bisphenol A oral reference dose (RfD) and drinking-water equivalent concentration. Journal of toxicology and environmental health. Part B, Critical reviews 11, 69-146, doi:10.1080/10937400701724303 (2008)

[2]. Roy, D. et al. Biochemical and molecular changes at the cellular level in response to exposure to environmental estrogen-like chemicals. Journal of toxicology and environmental health 50, 1-29 (1997).

[3]. Wiseman, H. \& Halliwell, B. Damage to DNA by reactive oxygen and nitrogen species: role in inflammatory disease and progression to cancer. The Biochemical journal 313 ( Pt 1), 17-29 (1996).

[4]. Kabuto, H. Effects of bisphenol A on the metabolisms of active oxygen species in mouse tissues. Environmental research 93, 31-35, doi:10.1016/s0013-9351(03)00062-8 (2003).

[5]. Ho, Y. S., Magnenat, J. L., Gargano, M. \& Cao, J. The nature of antioxidant defense mechanisms: a lesson from transgenic studies. Environmental health perspectives 106 Suppl 5, 1219-1228 (1998).

[6]. Bindhumol, V., Chitra, K. C. \& Mathur, P. P. Bisphenol A induces reactive oxygen species generation in the liver of male rats. Toxicology 188, 117-124, doi:10.1016/s0300-483x(03)00056-8 (2003)

[7]. Chitra, K. C., Latchoumycandane, C. \& Mathur, P. P. Induction of oxidative stress by bisphenol A in the epididymal sperm of rats. Toxicology 185, 119-127 (2003).

[8]. Hanioka, N., Jinno, H., Tanaka-Kagawa, T., Nishimura, T. \& Ando, M. Interaction of bisphenol A with rat hepatic cytochrome P450 enzymes. Chemosphere 41, 973-978 (2000).

[9]. Krahenbuhl, S. Mitochondria: important target for drug toxicity? Journal of hepatology 34, 334-336 (2001).

[10]. Qin, Z. et al. Role of Menkes ATPase in angiotensin II-induced hypertension: a key modulator for extracellular superoxide dismutase function. Hypertension 52, 945-951, doi:10.1161/HYPERTENSIONAHA.108.116467 (2008).

[11]. Fridovich, I. Superoxide anion radical (O2-.), superoxide dismutases, and related matters. The Journal of biological chemistry 272 , 18515-18517 (1997)

[12]. Di Naso, F. C., Simoes Dias, A., Porawski, M. \& Marroni, N. A. Exogenous superoxide dismutase: action on liver oxidative stress in animals with streptozotocin-induced diabetes. Experimental diabetes research 2011, 754132, doi:10.1155/2011/754132 (2011).

[13]. Yamasaki, K., Okuda, H., Takeuchi, T. \& Minobe, Y. Effects of in utero through lactational exposure to dicyclohexyl phthalate and p,p'-DDE in Sprague-Dawley rats. Toxicology letters 189, 14-20, doi:10.1016/j.toxlet.2009.04.023 (2009).

[14]. You, L. et al. Impaired male sexual development in perinatal Sprague-Dawley and Long-Evans hooded rats exposed in utero and lactationally to p,p'-DDE. Toxicological sciences : an official journal of the Society of Toxicology 45, 162-173 (1998).

[15]. Richter, C. A. et al. In vivo effects of bisphenol A in laboratory rodent studies. Reproductive toxicology 24, 199-224, doi:10.1016/j.reprotox.2007.06.004 (2007).

[16]. Pacher, P., Beckman, J. S. \& Liaudet, L. Nitric oxide and peroxynitrite in health and disease. Physiological reviews 87, 315-424, doi:10.1152/physrev.00029.2006 (2007)

[17]. MARKOVÁ, M., E. ADÁMEKOVÁ, P. KUBATKA, B. BOJKOVÁ, E. AHLERSOVÁ, I. AHLERS. Effect of prolonged melatonin administration on metabolic parameters and organ weights in young male and fenale Sprague-Dawley rats. ACTA VET. 72: 163-173. BRNO (2003).

[18]. Adachi, T. et al. Quantitative and qualitative changes of extracellular-superoxide dismutase in patients with various diseases. Clinica chimica acta; international journal of clinical chemistry 229, 123-131 (1994).

[19]. Vaziri, N. D., Ni, Z., Oveisi, F. \& Trnavsky-Hobbs, D. L. Effect of antioxidant therapy on blood pressure and NO synthase expression in hypertensive rats. Hypertension 36, 957-964 (2000).

[20]. Suzuki, H., Swei, A., Zweifach, B. W. \& Schmid-Schonbein, G. W. In vivo evidence for microvascular oxidative stress in spontaneously hypertensive rats. Hydroethidine microfluorography. Hypertension 25, 1083-1089 (1995).

[21]. Nakazono, K. et al. Does superoxide underlie the pathogenesis of hypertension? Proceedings of the National Academy of Sciences of the United States of America 88, 10045-10048 (1991).

[22]. Rajagopalan, S. et al. Angiotensin II-mediated hypertension in the rat increases vascular superoxide production via membrane NADH/NADPH oxidase activation. Contribution to alterations of vasomotor tone. The Journal of clinical investigation 97, 19161923, doi:10.1172/JCI118623 (1996)

[23]. Laursen, J. B. et al. Role of superoxide in angiotensin II-induced but not catecholamine-induced hypertension. Circulation 95, 588593 (1997).

[24]. Swei, A., Lacy, F., DeLano, F. A. \& Schmid-Schonbein, G. W. Oxidative stress in the Dahl hypertensive rat. Hypertension 30, 1628-1633 (1997)

[25]. Vaziri, N. D., Ding, Y. \& Ni, Z. Compensatory up-regulation of nitric-oxide synthase isoforms in lead-induced hypertension; reversal by a superoxide dismutase-mimetic drug. The Journal of pharmacology and experimental therapeutics 298, 679-685 (2001).

[26]. Gavella, M., Lipovac, V., Vucic, M. \& Rocic, B. Relationship of sperm superoxide dismutase-like activity with other spermspecific enzymes and experimentally induced lipid peroxidation in infertile men. Andrologia 28, 223-229 (1996).

[27]. Sinha, S., Pradeep, K. G., Laloraya, M. \& Warikoo, D. Over-expression of superoxide dismutase and lack of surface-thiols in spermatozoa: inherent defects in oligospermia. Biochemical and biophysical research communications 174, 510-517 (1991).

[28]. Jones, R., Mann, T. \& Sherins, R. Peroxidative breakdown of phospholipids in human spermatozoa, spermicidal properties of fatty acid peroxides, and protective action of seminal plasma. Fertility and sterility 31, 531-537 (1979).

[29]. McCord, J. M. The evolution of free radicals and oxidative stress. The American journal of medicine 108, $652-659$ (2000).

[30]. Oberley, L. W. Anticancer therapy by overexpression of superoxide dismutase. Antioxidants \& redox signaling 3, 461-472, doi:10.1089/15230860152409095 (2001)

[31]. Plymate, S. R. et al. Increased manganese superoxide dismutase (SOD-2) is part of the mechanism for prostate tumor suppression by Mac25/insulin-like growth factor binding-protein-related protein-1. Oncogene 22, 1024-1034, doi:10.1038/sj.onc.1206210 (2003).

[32]. Maneesh, M., Dutta, S., Chakrabarti, A. \& Vasudevan, D. M. Alcohol abuse-duration dependent decrease in plasma testosterone and antioxidants in males. Indian journal of physiology and pharmacology 50, 291-296 (2006).

[33]. Kim, G. W., Lewen, A., Copin, J., Watson, B. D. \& Chan, P. H. The cytosolic antioxidant, copper/zinc superoxide dismutase, attenuates blood-brain barrier disruption and oxidative cellular injury after photothrombotic cortical ischemia in mice. Neuroscience 105, 1007-1018 (2001).

[34]. Sprenger, C. C., Vail, M. E., Evans, K., Simurdak, J. \& Plymate, S. R. Over-expression of insulin-like growth factor binding protein-related protein-1(IGFBP-rP1/mac25) in the M12 prostate cancer cell line alters tumor growth by a delay in G1 and cyclin A associated apoptosis. Oncogene 21, 140-147, doi:10.1038/sj.onc.1205021 (2002). 
[35]. Touahar, I. E., Haroune, L., Ba, S., Bellenger, J. P. \& Cabana, H. Characterization of combined cross-linked enzyme aggregates from laccase, versatile peroxidase and glucose oxidase, and their utilization for the elimination of pharmaceuticals. The Science of the total environment 481C, 90-99, doi:10.1016/j.scitotenv.2014.01.132 (2014).

[36]. Boockfor, F. R. \& Blake, C. A. Chronic administration of 4-tert-octylphenol to adult male rats causes shrinkage of the testes and male accessory sex organs, disrupts spermatogenesis, and increases the incidence of sperm deformities. Biology of reproduction 57, 267-277 (1997).

[37]. Aydogan, M. \& Barlas, N. Effects of maternal 4-tert-octylphenol exposure on the reproductive tract of male rats at adulthood. Reproductive toxicology 22, 455-460, doi:10.1016/j.reprotox.2006.01.004 (2006).

[38]. Atkinson, A. \& Roy, D. In vitro conversion of environmental estrogenic chemical bisphenol A to DNA binding metabolite(s). Biochemical and biophysical research communications 210, 424-433, doi:10.1006/bbrc.1995.1678 (1995).

[39]. Suarez, S., Sueiro, R. A. \& Garrido, J. Genotoxicity of the coating lacquer on food cans, bisphenol A diglycidyl ether (BADGE), its hydrolysis products and a chlorohydrin of BADGE. Mutation research 470, 221-228 (2000). 